\title{
Comparison of the Effect of Calcium Hydroxide Nanoparticles Paste versus Double Antibiotic Paste Used in Regenerative Endodontic Procedure on the Chemical Structure of Radicular Dentin (An In Vitro study)
}

\author{
Mohamed Khaled Hassan Abd El Bary ${ }^{1 *}$, Jealan Mohamed El Shafei ${ }^{2}$ and Nehal Nabil Roshdy ${ }^{3}$ \\ ${ }^{1}$ Department of Endodontics, Faculty of Dentistry, Cairo University, Egypt \\ ${ }^{2}$ Professor of Endodontics, Faculty of Dentistry, Cairo University, Egypt \\ ${ }^{3}$ Lecturer in Endodontics, Faculty of Dentistry, Cairo University, Egypt \\ *Corresponding Author: Mohamed Khaled Hassan Abd El Bary, Master's degree Student, Department Of Endodontics, Faculty of Den- \\ tistry, Cairo University, Egypt.
}

Received: July 19, 2019; Published: July 31, 2019

DOI: 10.31080/ASDS.2019.03.0608

\begin{abstract}
Objective: The aim of the study was to compare the effect of calcium hydroxide nanoparticles paste versus double antibiotic pasteused in regenerative endodontic procedures on the chemical structure of radicular dentin.

Material and methods: Sixteen maxillary central incisors roots were sectioned to form 63 samples, then were divided to three equal groups (n=21); Group (1) Distilled water, Group (2) Calcium hydroxide nanoparticles and Group (3) Double antibiotic paste. Each group was further subdivided to 3 subgroups according to the time of measurement (1, 2 and 4 weeks). After each time point, each sample was examined using Fourier transformation infrared (FTIR) spectroscopy which analyzed the relative loss of organic and inorganic components by using phosphate/amide I ratios.

Results: Calcium hydroxide nanoparticles showed a statistically significant effect on phosphate/amide I ratios after 2 and 4 weeks. Double antibiotic paste showed a significant effect on phosphate/amide I ratios after 4 weeks.

Conclusion: Both calcium hydroxide nanoparticles and double antibiotic paste established a significant effect on the chemical structure of radicular dentin after 4 weeks application.
\end{abstract}

Keywords: Calcium Hydroxide Nanoparticles; Chemical Structure of Dentin; Double Antibiotic Paste; FTIR; Regenerative Endodontic Procedure

\section{Abbreviations}

TAP: Triple Antibiotic Paste; DAP: Double Antibiotic Paste; FTIR: Fourier Transform Infrared Spectroscopy; NaOCl: Sodium Hypochlorite.

\section{Introduction}

Management of necrotic immature teeth presents an endodontic challenge. Not only do necrotic immature teeth possess thin fragile dentin walls, but also a compromised crown root ratio [1]. For long 
time, apexification had been the treatment of choice for such cases, which resulted in formation of hard tissue barrier apically without completion of root development [2]. Recently, many clinical studies and case reports supported the use of endodontic regenerative procedure in the management of such cases which resulted in increasing the dentinal walls thickness as well as induction of the root formation [3].

Calcium hydroxide has long been used as an intracanal medicament. It has the ability to decrease pain because it neutralizes the acidity of bacterial byproducts through its alkaline PH [4]. On the other hand, antibiotic pastes have been used for disinfection of root canal system in regenerative endodontic procedure. The most commonly used paste is the triple antibiotic paste (TAP) which consists of Minocycline, Ciprofloxacin and Metronidazole [5]. However, Minocycline caused tooth discoloration, thus some authors suggested its elimination and keeping Metronidazole and ciprofloxacin to be used as double antibiotic paste (DAP) [6].

Antibiotic pastes showed higher antibacterial efficacy in bacterial eradication than calcium hydroxide [7]. On the other hand, the introduction of nanotechnology in endodontic dentistry, developed new materials like Calcium hydroxide nanoparticles that show promising antibacterial effect with high penetration power and same cytotoxicity than conventional calcium hydroxide $[8,9]$.

However, the use of Intracanal medicaments had a negative impact on the chemical structure of radicular dentin causing reduction in its mechanical properties [10]. Thereby, the proper selection of intracanal medication is crucial. There were no previous studies assessing the effect of double antibiotic paste and Calcium hydroxide nanoparticles paste on the chemical structure of radicular dentin.

The aim of this study was to compare the effect of calcium hydroxide nanoparticles paste versus double antibiotic paste (ciprofloxacin and metronidazole) on the chemical structure of radicular dentin using Fourier Transformation Infrared (FTIR) Spectroscopy.

The null hypothesis was that there would be no statistically significant difference between calcium hydroxide nanoparticles paste and double antibiotic paste on the chemical structure of radicular dentin

\section{Materials and Methods}

Sample size calculation

Based on a previous study performed by Yassen., et al [5], an estimated large effect size of approximately 0.519 was used. Considering that the outcome variable was not normally distributed using the power of $80 \%$, and a significance level of $5 \%$, a total sample size of 50 was sufficient. The umber was increased to 63 (21 samples per group and 7 samples per subgroups) to account for the necessity of using non-parametric test. Sample size was calculated using G*Power program (University of Düsseldorf, Düsseldorf, Germany).

\section{Selection of samples}

Sixteen human permanent maxillary central incisors extracted for periodontal reasons were collected from the oral and maxillofacial department, Faculty of dentistry, Cairo University. Initial radiographs were taken to ensure that all teeth had mature apices, with no internal or external resorption, calcifications or root caries. The collected teeth were cleaned from any hard and soft deposits by using an ultrasonic scaler and were disinfected in $2.6 \%$ sodium hypochlorite for 2-3 hours. Then, teeth were restored in saline solution until use.

\section{Preparation of samples}

Each tooth was mounted in an acrylic resin block using core buildup material. Each acrylic resin block was mounted on laser precision saw (Isomet 4000, Buehler, Lake Bluff, IL, USA). The crowns were removed at the cementoenamel and the roots were leveled off (flattened) to form a flat surface on each side using a water-cooled low-speed diamond saw. Each root was sectioned longitudinally then horizontally to form $4 \mathrm{~mm} \times 4 \mathrm{~mm}$ specimens (Each root comprised 4 specimens). The specimens were rinsed for 5 minutes with distilled water with 25G needle (Ameco, Tenth of Ramadan City, Egypt) and ultasonicated for extra 5 minutes to remove debris which were developed during cutting procedure. Sixteen teeth formed 64 samples in which one of them was randomly discarded to obtain 63 samples.

\section{Preparation of intracanal medicament}

Calcium hydroxide nanoparticles was mixed with distilled water to form creamy mix (1gram powder to $1 \mathrm{ml}$ distilled water). While, the double antibiotic paste was prepared by grinding an equal 
number of tablets of Flagyl 500mg and Ciprofloxacin 500mg. The powder was then mixed with distilled water to form creamy mix (1gram powder to $1 \mathrm{ml}$ distilled water). The pastes were loaded in $3 \mathrm{~cm}$ syringe.

Each tooth sample was placed in $1.5 \mathrm{~mL}$ Eppendorf and covered with $0.5 \mathrm{~mL}$ of prepared intracanal medication pastes. The medicaments were injected in the Eppendorf by using the modified plastic syringe and applying slight tapping to remove entrapped air voids. For control group distilled water was applied by $25 \mathrm{G}$ needle.

\section{FTIR analysis}

For measuring the change in the chemical structure, each sample was rinsed with distilled water for 5 minutes with needle gauge size \# 25 till no medicament became visible and ultrasonicated for 5 minutes. Each sample was positioned vertically and scratched with (No 15) blade to form dentin powder. The powder was mixed with Potassium Bromide powder and was compressed as well by hydraulic machine between two solid potassium bromide laminas to form a tablet. Fourier transformation infrared (FTIR) spectroscopy (JASCO INTERNATIONAL CO., LTD.) was used to measure the absorbance of infrared spectra for analyzing radicular dentin chemical structure and from the results (phosphate/amide I) ratio was measured which represent loss of organic and inorganic structures.

FTIR spectra were then collected from the 3 selected areas in each tablet between 800 and 2,000 cm-1 at $4 \mathrm{~cm}-1$ resolution. Each spectrum was then processed using Spectra manager software. The effect of various treatments on collagen and apatite composition of dentin was evaluated by using the mineral matrix ratio (the ratio of integrated areas of the phosphate contour to the amide I peak). Larger ratios corresponded to a greater dentin collagen deproteinization, while smaller ratios corresponded to a greater dentin demineralization.

\section{Statistical analysis}

Numerical data were explored for normality by checking the distribution of the data and using tests of normality (KolmogorovSmirnov and Shapiro-Wilk tests). All data showed normal (parametric) distribution. Data were presented as mean, median, standard deviation (SD), minimum, maximum and 95\% Confidence Interval $(95 \% \mathrm{CI})$ for the mean values.
Two-way Analysis of Variance (ANOVA) was used to study the effect of group, storage time and their interaction on mean P04/ Amide I ratio. Bonferroni's post-hoc test was used for pair-wise comparisons when ANOVA test is significant.

The significance level was set at $\mathrm{P} \leq 0.05$. Statistical analysis was performed with IBM® SPSS $®$ statistics version 20 for windows.

\section{Results}

Group 1 (control)

Our results showed that the mean values of P04/Amide I ratio were 1.78, 1.75and 1.78 at 1,2 and 4 weeks respectively. There was no statistically significant difference among the 3 subgroups $(\mathrm{P}=0.980)$.

\section{Group 2 (Calcium hydroxide nanoparticles)}

Our results showed that the mean values of P04/Amide I ratio were $1.85,2.45$ and 2.66 at 1,2 and 4 weeks respectively. There was a statistically significant increasing between 1 week subgroup and 2 and 4 weeks' subgroups. Two and 4 weeks subgroups showed no statistical significant difference between them $(\mathrm{P}<0.001)$.

\section{Group 3 (DAP)}

Our results showed that the mean values of P04/Amide I ratio were $1.69,1.92$ and 2.29 at 1, 2 and 4 weeks respectively. There was no statistically significant difference between 1 week and 2 week subgroups but there was significant increasing in 4 weeks subgroup $(\mathrm{P}<0.003)$.

All data are presented in Table 1 and 2, Figure 1.

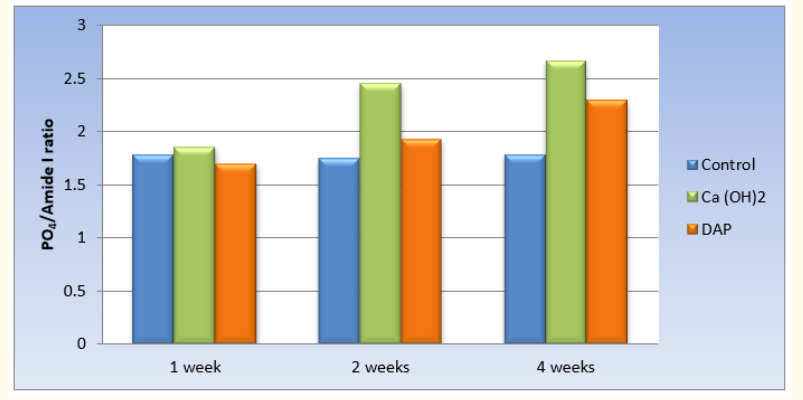

Figure 1: Bar chart representing mean values for P04/Amide I ratio of the different interactions. 
Comparison of the Effect of Calcium Hydroxide Nanoparticles Paste versus Double Antibiotic Paste Used in Regenerative Endodontic Procedure on the Chemical Structure of Radicular Dentin (An In Vitro study)

\begin{tabular}{|c|c|c|c|c|c|c|c|c|}
\hline \multirow[b]{2}{*}{ Group } & \multirow{2}{*}{$\begin{array}{l}\text { Storage } \\
\text { time }\end{array}$} & \multirow[b]{2}{*}{ Mean } & \multirow[b]{2}{*}{ SD } & \multirow[b]{2}{*}{ Median } & \multirow[b]{2}{*}{ Minimum } & \multirow[b]{2}{*}{ Maximum } & \multicolumn{2}{|c|}{ 95\% CI } \\
\hline & & & & & & & $\begin{array}{l}\text { Lower } \\
\text { bound }\end{array}$ & $\begin{array}{l}\text { Upper } \\
\text { bound }\end{array}$ \\
\hline \multirow[t]{3}{*}{ Control } & 1 week & 1.78 & 0.16 & 1.76 & 1.60 & 2.05 & 1.63 & 1.92 \\
\hline & 2 weeks & 1.75 & 0.26 & 1.77 & 1.40 & 2.24 & 1.51 & 2.00 \\
\hline & 4 weeks & 1.78 & 0.32 & 1.77 & 1.47 & 2.42 & 1.49 & 2.08 \\
\hline \multirow{3}{*}{ nanoparticles } & 1 week & 1.85 & 0.28 & 1.76 & 1.52 & 2.34 & 1.59 & 2.10 \\
\hline & 2 weeks & 2.45 & 0.37 & 2.56 & 2.03 & 3.00 & 2.11 & 2.80 \\
\hline & 4 weeks & 2.66 & 0.35 & 2.55 & 2.36 & 3.37 & 2.33 & 2.98 \\
\hline \multirow[t]{3}{*}{ DAP } & 1 week & 1.69 & 0.40 & 1.53 & 1.31 & 2.32 & 1.32 & 2.06 \\
\hline & 2 weeks & 1.92 & 0.22 & 2.03 & 1.54 & 2.13 & 1.72 & 2.13 \\
\hline & 4 weeks & 2.29 & 0.34 & 2.18 & 1.90 & 2.84 & 1.97 & 2.60 \\
\hline
\end{tabular}

Table 1: Descriptive statistics for $\mathrm{PO}_{4} /$ Amide I ratio values of the different groups at different observation periods.

Comparison between the three tested groups

At 1 week

Comparison between distilled water group, Calcium hydroxide nanoparticles group and DAP group after 1 week showed no statistically significant difference among the 3 groups $(\mathrm{P}=0.653)$.

At 2 weeks

1. Comparison between distilled water group and Calcium hydroxide nanoparticles group showed a statistically significant difference $(\mathrm{P}<0.001)$.

2. Comparison between distilled water group and DAP showed no statistical significant difference.
3. Comparison between Calcium hydroxide nanoparticles group and DAP group showed a statistically significant difference.

At 4 weeks

1. Comparison between distilled water group and Calcium hydroxide nanoparticles group showed a statistically significant difference $(\mathrm{P}<0.001)$.

2. Comparison between distilled water group and DAP group showed at a statistically significant difference.

3. Comparison between Calcium hydroxide nanoparticles group and DAP group showed statistically significant difference.

All data are presented in Table 2

\begin{tabular}{|c|c|c|c|c|c|c|c|}
\hline \multirow{2}{*}{ Storage time } & \multicolumn{2}{|c|}{ Control } & \multicolumn{2}{|c|}{$\begin{array}{c}\mathrm{Ca}(\mathrm{OH})_{2} \\
\text { nanoparticles }\end{array}$} & \multicolumn{2}{|c|}{ DAP } & \multirow{2}{*}{$\begin{array}{c}\text { P-value } \\
\text { (Between groups) }\end{array}$} \\
\hline & Mean & SD & Mean & SD & Mean & SD & \\
\hline 1 week & 1.78 & 0.16 & $1.85^{\mathrm{E}}$ & 0.28 & $1.69^{\mathrm{E}}$ & 0.40 & 0.653 \\
\hline 2 weeks & $1.75^{\text {в }}$ & 0.26 & $2.45^{\mathrm{AD}}$ & 0.37 & $1.92^{\mathrm{BE}}$ & 0.22 & $<0.001^{*}$ \\
\hline 4 weeks & $1.78^{\mathrm{C}}$ & 0.32 & $2.66^{\mathrm{AD}}$ & 0.35 & $2.29^{\mathrm{BD}}$ & 0.34 & $<0.001^{*}$ \\
\hline P-value (Between storage times) & \multicolumn{2}{|c|}{0.980} & \multicolumn{2}{|c|}{$<0.001^{*}$} & \multicolumn{2}{|c|}{$0.003^{*}$} & \\
\hline
\end{tabular}

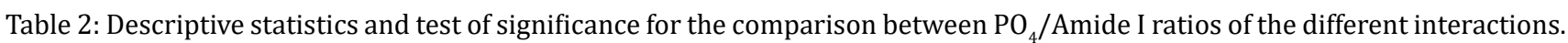

*: Significant at $\mathrm{P} \leq 0.05$

A, B, C superscripts in the same row indicate significant differences between groups

D, E, F superscripts in the same column indicate significant differences between storage times 
Comparison of the Effect of Calcium Hydroxide Nanoparticles Paste versus Double Antibiotic Paste Used in Regenerative Endodontic Procedure on the Chemical Structure of Radicular Dentin (An In Vitro study)

Effect of group regardless of storage time in P04/Amide I ratio

Regardless of storage time, Calcium hydroxide nanoparticles group showed the statistically significantly highest mean P04/ Amide I ratio. There was no statistically significant difference between control and DAP groups; both showed the statistically significantly lowest mean $\mathrm{PO}_{4} /$ Amide I ratios Table 3 .

\begin{tabular}{|l|l|c|c|c|c|c|}
\hline \multicolumn{2}{|l|}{ Control } & \multicolumn{2}{|c|}{$\begin{array}{c}\text { Ca (OH) } \\
\text { nanoparticles }\end{array}$} & \multicolumn{2}{c|}{ DAP } & \multirow{2}{*}{ P-value } \\
\cline { 1 - 5 } Mean & SD & Mean & SD & Mean & SD & \\
\hline $1.77^{\text {В }}$ & 0.24 & $2.32^{\text {A }}$ & 0.448 & $1.97^{\text {В }}$ & 0.40 & $<0.001^{*}$ \\
\hline
\end{tabular}

Table 3: Descriptive statistics and test of significance for the comparison between $\mathrm{PO}_{4} /$ Amide I ratios of the three groups regardless of storage time.

*: Significant at P $\leq 0.05$, Different superscripts in the same row are statistically significantly different

Effect of storage time regardless of group in P04/Amide I ratio

Regardless of group, the mean $\mathrm{PO}_{4} /$ Amide I ratio after 1 week showed the statistically significantly lowest mean value. There was no statistically significant difference between mean $\mathrm{PO}_{4} /$ Amide I ratio after 2 weeks and 4 weeks; both showed statistically significantly higher mean ratios Table 4

\begin{tabular}{|c|c|c|c|c|c|c|}
\hline \multicolumn{2}{|c|}{ 1 week } & \multicolumn{2}{c|}{ 2 weeks } & \multicolumn{2}{c|}{ 4 weeks } & \multirow{2}{*}{ P-value } \\
\cline { 1 - 5 } Mean & SD & Mean & SD & Mean & SD & \\
\hline $1.77^{\text {B }}$ & 0.29 & $2.04^{\mathrm{A}}$ & 0.41 & $2.24^{\mathrm{A}}$ & 0.49 & \multirow{2}{*}{$<0.001^{*}$} \\
\hline
\end{tabular}

Table 4: Descriptive statistics and test of significance for the comparison between $\mathrm{PO}_{4} /$ Amide I ratios at different storage times regardless of group.

*: Significant at $\mathrm{P} \leq 0.05$, Different superscripts in the same row are statistically significantly different.

\section{Two-way ANOVA results}

The results showed that group, storage time and the interaction between the two variables had a statistically significant effect on mean $\mathrm{PO}_{4} /$ Amide I ratio. Since the interaction between the variables is statistically significant, so the variables are dependent upon each other Table 5.

\begin{tabular}{|l|c|c|c|c|c|c|}
\hline Source of variation & $\begin{array}{c}\text { Type III Sum of } \\
\text { Squares }\end{array}$ & df & Mean Square & F-value & $\begin{array}{c}\text { P-value } \\
\text { Partial Eta Squared } \\
\text { (Effect size) }\end{array}$ \\
\hline Group & 3.229 & 2 & 1.615 & 16.908 & $<0.001^{*}$ & 0.385 \\
\hline Storage time & 2.338 & 2 & 1.169 & 12.239 & $<0.001^{*}$ & 0.312 \\
\hline Group x Storage time interaction & 1.414 & 4 & 0.353 & 3.701 & $0.010^{*}$ & 0.215 \\
\hline
\end{tabular}

Table 5: Two-way ANOVA results for the effect of different variables on mean $\mathrm{PO}_{4} /$ Amide I ratio

df: degrees of freedom $=(n-1)$,

*: Significant at $\mathrm{P} \leq 0.05$

\section{Discussion}

Treating immature necrotic teeth has remained a challenging situation for years. Since, it is very difficult to establish proper apical seal as they lack apical constriction together with their high liability to fracture owing to the thin dentinal walls and short roots. In 1961, Nygaard-Ostby introduced the concept of regenerative endodontic therapy by trying to recreate tissues in the apical area of the root canal in mature teeth [10]. Then, in 2004 Banchs and Trope reintroduced the concept to modern endodontics in immature teeth by disinfecting the canal, then establish bleeding and final sealing with MTA [11].
Since, the key procedure of the regenerative endodontic treatment is either minimal or no instrumentation of the dentinal walls of the root canal, thereby, effective elimination of bacteria present inside root canal relies mainly upon proper disinfection. Disinfection is established by gentle, thorough irrigation and placement of potent antibacterial intracanal medicament [11]. However, intracanal medicaments showed some drawbacks such as cytotoxcicty, teeth discoloration, and increase risk of root fracture in immature teeth [12-14]. 
Calcium hydroxide nanoparticles was used in this study to test its effect on the chemical structure of radicular dentin. In 2016 the American Association of Endodontists (AAE) has included Calcium hydroxide as an alternative intracanal medicament for regenerative endodontics [15]. The material has been proved not to cause staining on the tooth structure and allow the release of growth factors and biomolecules from dentin structure [16]. The main drawback was the low antimicrobial effect of Calcium hydroxide than double antibiotic paste against bacterial biofilm obtained from mature and immature teeth with Necrotic Pulp [17].The introduction of Calcium hydroxide nanoparticles to dental field prove to possess higher antibacterial effect than conventional Calcium hydroxide with similar cytotoxic effect and believed to show promising results in dental field [18]. Since, nanotechnology deals with atoms; it provided the manipulation of different materials at nanoscale for different purposes. Since, dealing with materials in nanoscales has many advantages such as high reactivity and large surface area in small volume of material that allow faster and stronger action [19].

The conventional Calcium hydroxide particles has a diameter range of $2.255 \pm 1.994(\mu \mathrm{m})$ in length and $1.620 \pm 1.464(\mu \mathrm{m})$ in width which diameter may allow the penetration of Calcium hydroxide inside some dentinal tubules but Calcium hydroxide nanoparticles have particles size ranging from 10 to $200 \mathrm{~nm}$ which allow more powerful penetration inside dentinal tubules(2 -5 $\mu \mathrm{m}$ diameter) that give more effect $[20,21]$.

Double antibiotic paste was selected in our study because it is commonly used as an intracanal medication in regenerative endodontic therapy in anterior teeth to avoid the staining caused by the minocycline present in the triple antibiotic paste [22]. Minocycline has high cytotoxic effect on human cells that affect survival of SCAPs [23]. Also triple antibiotic paste has highly acidic PH (2.9) that affects the microhardness of dentin [24] which was formed from the commercially available tablets (ciprofloxacin 500 and flagyl 500) was selected because it is commonly used in clinical situation rather than DAP formed in lab from raw material, and its low cost. Nikita., et al. demonstrated that the Calcium hydroxide as an intracanal medicament in comparison to DAP showed less detrimental effect on SCAPs [12].
The mineral component of dentin is characterized essentially as nonstoichiometric substituted apatite, where the phosphate or carbonate spectral peaks are considered the main representative of this phase. The dentin organic phases are mainly composed of collagen type I that appears as spectral bands of amide I, II, and III bands [25]. Fourier transformation infrared spectroscope is considered ideal in studying chemical structural properties of natural materials, because the frequencies of several vibrational modes of inorganic and organic molecules are active in the infrared beams [26]. FTIR imaging technique is label-free technology to eliminate any possible interference within the studied systems [27]. FTIR is a powerful method for collecting direct information about the chemical structure of dentin and indirectly, about their molecular structure [25].

Upper anterior teeth were selected because they have conical roots which are easy in sectioning and give samples of high quality. The selected teeth were mature with closed apex because it is unethical to extract immature teeth. The use of laser precision saw gave smooth, flat and accurate dimension samples. Also, Laser precision saw allowed perfect cooling to samples during cutting which prevented heat generation that destroy organic component of the tooth structure [5].

Ultrasonic cleaning of samples was performed to remove debris from the surface of samples to allow for a better contact of intracanal medicament with sample surface [5].

Dentin samples were collected by scratching the surface of each sample, this method was selected rather than direct reading of sample surface because FTIR machine needs a very smooth surface and it is very difficult to be achieved in biological samples [27]. Also scratching not only gives an idea about the changes occurring superficially but also those occurring in the core because penetration depth of FTIR beams limited to few microns of dentin surface and scratching give an idea about the deeper layers [28].

According to AAE, the clinical considerations for a regenerative procedure (2016); time interval between first and second appointment varies from one to four weeks. Where, some cases showed success after 1 week [29]. On the other hand, a 2- to 4-week treatment period is considered sufficient to obtain a therapeutic effect $[30,31]$, So the chosen time period for this study was 1, 2 and 4 weeks as time points. 
In our study Calcium hydroxide nanoparticles showed the highest statistically significantly mean P04/Amide I ratio after 2 and 4 time points. These results support the proposed denaturation effect of highly alkaline Calcium hydroxide $(\mathrm{pH}=11.8)$ on dentin organic matrix [5,32]. Where, small molecular weight of Calcium hydroxide particles (56.1 Da) facilitate its penetration through the apatite-encapsulated collagen matrix and lead to change in the 3-dimensional conformation of tropocollagen $[5,33]$.

There was no statistically significant difference between Calcium hydroxide nanoparticles and the control group after 1 week, but there was difference between 1 week and after 2, 4 weeks; the latter showed higher statistically significant mean ratios. Though, Yassen., et al. 2013 measured the effect of Calcium hydroxide on chemical structure of dentin and showed that there was significant increase in phosphate amide I ratio after 1,2,4 weeks with no statistical significant difference between them [5]. The difference could be attributed to the difference in the reading method where they used attenuated total reflection FTIR that gave reading on the surface of dentin and did not give an idea about the core [35], However, the difference between 2 weeks and 4 weeks was not significant and that could be due to high reactivity and surface energy of nanoparticles materials that lead to fast effect in short period of time. On the contrary of our results, Yassen., et al, 2015 measured the effect of Calcium hydroxide on the chemical structure of dentin after 4 weeks and showed significant decrease in phosphate amide ratio, which could be endorsed to the different irrigation protocol, where they used $20 \mathrm{~mL}$ of $1.5 \% \mathrm{NaOCl}$ for 5 minutes before the application of Calcium hydroxide and $20 \mathrm{~mL}$ of $17 \%$ EDTA for 10 minutes after one month. Where, $\mathrm{NaOCl}$ and EDTA had affected the chemical structure of dentin, but in our study only distilled water was used before application of calcium hydroxide and after each time period [28].

On the other hand, DAP $(\mathrm{pH}=3.4)$ is an acidic material that have a demineralization effect on the dental hard tissues causing exposure of collagen-rich matrix on the outer surface of the radicular dentin, which was detected by the scanning electric microscope [5]. Our study showed that the phosphate/amide I ratio was significantly higher at 4-week time period when compared to 1- and 2-weeks treated dentin, with no statistically significant difference between control, 1-week and 2-week time periods which indicate gradual effect of DAP. Unlike, Yassen., et al, 2013 that showed double antibiotic paste decreased the phosphate/amide I ratio after 1, 2 and 4 weeks [5].

The difference in results could be explained by the chemical overlapping in the measured spectra between the residues of organic C-O-C peak from methylcellulose which is present in the composition of antibiotic tablets and the inorganic phosphate peaks of radicular dentine. This theory is strongly supported by other study which compared the effect of triple antibiotic paste and triple antibiotic paste mixed with methyl cellulose at different concentrations on the chemical structure of dentin [34]. Additionally, Methylcellulose leads to retention of intracanal medicaments when used as vehicle as it resists dissolution and removal of Calcium hydroxide by the aqueous irrigation solutions which may affect reading [35].

Our results showed that both Calcium hydroxide nanoparticles and double antibiotic paste had a significant effect on the chemical structure of dentin after 4 weeks which can be correlated to the effect of intracanal medicament on the microhardness of dentin, due to either collagen degradation with Calcium hydroxide or excessive demineralization with double antibiotic pastes.

Nevertheless, double antibiotic paste showed a stronger effect on microhardness of dentin in comparison to Calcium hydroxide [24]. This could be related to high acidity of double antibiotic paste which leads to continuous action over long time.

Other laboratory study concluded that long term application of Calcium hydroxide and double antibiotic pastes significantly reduced the root fracture resistance on extracted teeth [5]. Even though, there is no clinical evidence to show that microhardness is related to root fractures, that effect might reduce the fracture resistance and cause propagation of dentinal microcracks which might lead to vertical root fractures [24].

The demineralization effect of the double antibiotic paste plays an important role in pulp regeneration by enhancing the stem cells attachment by exposing dentin collagen $[5,36]$.

\section{Conclusion}

Within the limitation of this study, it can be concluded that calcium hydroxide nanoparticles and double antibiotic paste established a significant effect on the chemical structure of radicular 
dentin either by collagen degradation or demineralization after 4 weeks application. However, double antibiotic paste showed a less destructive effect on the chemical structure of dentin after 2 weeks.

\section{Bibliography}

1. Silva M., et al. "Revascularization of an Immature Tooth with Apical Periodontitis Using Calcium Hydroxide: A 3-Year Follow-Up". The Open Dentistry Journal 9 (2015): 482-485.

2. Nicoloso G. "A comparative evaluation of endodontic treatments for immature necrotic permanent teeth based on clinical and radiographic outcomes: a systematic review and meta-analysis". International Journal of Paediatric Dentistry 27.3 (2017): 217-227.

3. Antunes L., et al. "The effectiveness of pulp revascularization in root formation of necrotic immature permanent teeth: A systematic review". Acta Odontologica Scandinavica 74.3 (2015) 161-169.

4. De Rossi A. "Effect of rotary or manual instrumentation, with or without a calcium hydroxide $/ 1 \%$ chlorhexidine intracanal dressing on the healing of experimentally induced chronic periapical lesions". Oral Surgery, Oral Medicine, Oral Pathology, Oral Radiology, and Endodontology 99.5 (2005): 628-636.

5. Yassen GH., et al. "Effect of Medicaments Used in Endodontic Regeneration Technique on the Chemical Structure of Human Immature Radicular Dentin : An In Vitro Study". Journal of Endodontics 39.2 (2013): 269-273.

6. Valverde ME., et al. "Antibacterial efficacy of several intracanal medicaments for endodontic therapy". Dental Materials Journal 36.3 (2017) 319-324.

7. Mehta S., et al. "Comparative evaluation of antimicrobial efficacy of triple antibiotic paste, calcium hydroxide, and a proton pump inhibitor against resistant root canal pathogens". European Journal of Dentistry 11.1 (2017): 53-75.

8. Dianat 0., et al. "Antimicrobial Activity of Nanoparticle Calcium Hydroxide against Enterococcus Faecalis: An In Vitro Study". Iranian endodontic journal 10.1 (2015): 39-43.

9. Dianat 0., et al. "Toxicity of calcium hydroxide nanoparticles on murine fibroblast cell line". Iranian endodontic journal 10.1 (2015): 49-54.

10. Ostby BN., et al. "The role of blood clot in endodontic therapy". Acta Odontologica Scandinavica 19.3 (1961): 323-353.
11. Banchs F and Trope M. "Revascularization of Immature Permanent Teeth with Apical Periodontitis : New Treatment Protocol ?". Journal of Endodontics 30.4 (2004): 196-200.

12. Nikita BR., et al. "Direct effect of intracanal medicaments on survival of stem cells of the apical papilla". Journal of Endodntics 38.10 (2012): 1372-1375.

13. Akcay M., et al. "Spectrophotometric analysis of crown discoloration induced by various antibiotic pastes used in revascularization". Journal of Endodontics 40.6 (2014): 845-848.

14. Yassen GH,. et al. "The effect of medicaments used in endodontic regeneration on root fracture and microhardness of radicular dentine". International Endodontic Journal 46.7 (2013) 688-695.

15. AAE: Clinical Considerations for a Regenerative Procedure (2016).

16. Graham L., et al. "The effect of Calcium hydroxide on solubilisation of bio-active dentine matrix components". Biomaterials 27.14 (2006): 2865-2873.

17. Jacobs JC., et al. "Antibacterial Effects of Antimicrobials Used in Regenerative Endodontics against Biofilm Bacteria Obtained from Mature and Immature Teeth with Necrotic Pulps". Journal of endodontics 43.4 (2017) :575-579.

18. Javidi M., et al. "Efficacy of a combined nanoparticulate/Calcium hydroxide root canal medication on elimination of Enterococcus faecalis". Austeralian Endodonticournal 40.2 (2013): 61-65.

19. Grumezescu AM. Nanobiomaterials in Dentistry Applications of Nanobiomaterials. (A. M. Grumezescu, Ed.) (1st ed.). Romania: Elsevier Inc,2016.

20. Biswas A., et al. "Advances in top-down and bottom-up surface nanofabrication: Techniques, applications and future prospects". Advances in Colloid and Interface Science 170.1-2 (2012): 2-27.

21. Komabayashi T., et al. "Particle Size and Shape of Calcium Hydroxide". Journal of Endodontics 35.2 (2009): 284-287.

22. Kahler B and Rossi-Fedele G. "A review of tooth discoloration after regenerative endodontic therapy". Journal of Endodontics 22.4 (2016): 563-569. 
23. Chuensombat S., et al. "Cytotoxic Effects and Antibacterial Efficacy of a 3-Antibiotic Combination : An In Vitro Study". Journal of Endodontics 39.18 (2013): 813-819.

24. Yilmaz S and Dumani A. "The effect of antibiotic pastes on microhardness of dentin". Dental Traumatolgy 32.21 (2016): 27-31.

25. Carvalho C De., et al. "Fourier transform infrared spectroscopy (FTIR) application chemical characterization of enamel , dentin and bone". Applied Spectroscopy Reviews 53.9 (2018): 747769.

26. Chan KLA and Kazarian SG. "Attenuated total reflection Fourier-transform infrared (ATR-FTIR) imaging of tissues and live cells". Chemal Society Reviews 45.7 (2015): 1850-1864.

27. Kazarian SG and Chan K. "Attenuated. ATR-FTIR spectroscopic imaging: recent advances and applications to biological systems Infrared". The Analyst 138.7 (2013): 1940-1951.

28. Yassen GH., et al. "Effect of intracanal medicaments used in endodontic regeneration procedures on microhardness and chemical structure of dentin". Restorative Dentistry and Endodntics 40.2 (2015): 104-112.

29. Jung I., et al. "Biologically Based Treatment of Immature Permanent Teeth with Pulpal Necrosis : A Case Series". Journal of Enododontics 34.7 ( 2008): 867-887.

30. Cehreli ZC., et al. "Regenerative Endodontic Treatment ( Revascularization ) of Immature Necrotic Molars Medicated with Calcium Hydroxide: A Case Series". Journal of Endodontics 37.9 (2011): 1327-1330.

31. Chen M., et al. "Responses of immature permanent teeth with infected necrotic pulp tissue and apical periodontitis/abscess to revascularization procedures". International Endodontic Journal 45.3 (2012): 294-305.

32. White JD., et al. "The Effect of Three Commonly Used Endodontic Materials on the Strength and Hardness of Root Dentin". Journal of Endodontics 28.14 (2002): 15-17.

33. Leiendecker AP., et al. "Effects of Calcium Silicate - based Materials on Collagen Matrix Integrity of Mineralized Dentin”. Journal of Endodontics 38.6 (2012): 829-833.
34. Prather BT., et al. "Effects of two combinations of triple antibiotic paste used in endodontic regeneration on root microhardness and chemical structure of radicular dentine". Journal of oral science 56.4 (2014): 245-251.

35. Lambrianidis T., et al. "Removal Efficiency of Calcium Hydroxide Dressing from the Root Canal”. Journal of Endodontics 25.2 (1999): 85-88.

36. Galler KM., et al. "Dentin Conditioning Codetermines Cell Fate in Regenerative Endodontics". Journal of Endodontics 37.11 (2011): 1536-1541.

\section{Volume 3 Issue 8 August 2019}

(C) All rights are reserved by Mohamed Khaled Hassan Abd El Bary., et al. 